Dr. Garrod proposes, that in cases of poisoning we should remove as much of the poison as possible by means of the stomach-pump or emetics, and then give a large quantity of the animal charcoal diffused in warm water, or the antidote may be given with the emetic, but ipecacuanha must not be used, as the charcoal would destroy its emetic property. Sulphate of zinc, or some other mineral emetic, should be chosen. Dr. Garrod also suggests that perhaps animal charcoal would prevent the action of the poison of rabies, syphilis, serpents, \&c., if applied in the form of a poultice to the part which has come into contact with the poison, and that it may prove serviceable as a remedy in some diseases, from its great power of absorbing all principles.-Pharm. Jour.

\title{
TUBERCULAR PHTHISIS, WITH EXTENSIVE ULCERATION OF THE
} LARYNX.

By John L. Vandervoort, M.D., of New York.

Miss —, aged 28 years, was attacked in July, 1845, with cough of an ordinary kind, coming on at intervals, either at night or early in the morning, and without expectoration. After continuing in this way for six or eight weeks, symptoms of difficulty in the larynx began to manifest themselves; these at first were very obscure, but after attentively watching the case, the conviction was strong in my mind that ulceration of the cartilages and membrane lining that organ was going on. The patient was of a peculiarly nervous temperament, very liable to violent paroxysms of hysteria; this gave rise to the suspicion that the cough was of this character, it being of a dry, hacking nature. This opinion was at first entertained by myself, and was coincided in by a gentleman who saw the paitent in consultation. Various means were resorted to for her relief, such as expectorants, emetics, tonics, mercurials, rubefacients, anodynes, blisters with morphine sprinkled on the raw surface, and the local application of a strong solution of nitrate of silver. No obvious relief following the employment of these remedies, and the aspect of the patient having undergone considerable change, together with slight emaciation, led to the suspicion of the existence of tubercles in one or both lungs. This opinion was confirmed by an examination of the chest, which was made by Dr. Swett, who detected the existence of tubercles in the sunmit of the right lung.

The disease of the lungs continued to progress unattended by any peculiar phenomena ; not so, however, that of the larynx. The ulceration of the membrane and cartilages of this organ, progressed from month to month, and gave rise not only to a very distressing cough, and intense dyspnœa, but to very great difficulty and pain in swallowing, especially liquids. For about two months prior to her death, she scarcely took anything but the blandest liquids, and these passing through the ulcerated openings into the trachea, often occasioned a vast amount of suffering.

About three days before her death, which tonk place the last of July, she expectorated pus quite freely; during this period her breathing was much easier and deglutition less painful. 
An examination of the body was made six hours after death. The following was the condition of the lungs and larynx:

The lungs were completely studded with tubercles in various stages; it the summit of the right lung was an abscess capable of holding about eight ounces; considerable serum was found in the chest.

The liver was of enormous size and of a mottled color; the left lobe a little softened; other abdominal organs healthy.

The larynx presented a mass of disease; the investing membrane was studded with granules of variable size, some of them being nearly as large as a split pea; by the process of ulceration it was completely detached from the cricoid cartilages, and could, by the means of a probe, be entirely raised from these bodies. By the side of these cartilages were ulcers of the size of a five-cent piece, which comınunicated with the cesophayus. The connecting ligarnents were gone, except one which was much thickened. The epiglottis was much thickened, and the arytenoid cartilages were nearly destroyed by ulceration.-The Annalist.

APOPLEXY FROM 'THE RUPTURE OF AN ANEURISM OF THE ARTERIA CEREHRI MEDIA.

By E. M. Fodder, C.M., Toronto.

Master H., æt. 10 years, fair complexion and highly nervous temperainent, received a severe shock at about half past 8 o'clock, P. M., on the 4 th of November last, in consequence of a fire, which at the moment was supposed to be in the building in which his father had his offices. The child had always been observed to become highly nerrous whenever the alarm of fire was given. He had a largely-developed bead, pale countenance, a somewhat delicate constitution, and generally depraved appetite, preferring crude vegetables and unripe fruits to more wholesome food.

In consequence of this, he suffered occasionally from derangement of the stomach and bowels, always attended with severe headache; but an occasional emetic and purgatives relieved him in a day or two.

Three or four weeks prior to the present date, he had had an attack as above described, during which time he complained very much of his head, but for the last fortnight he had appeared in perfect health.

On the alarm of fire being given, he ran into the street, but returned immediately to the house and watched the progress of the flames from a bed-room window; in three or four minutes lse gave a sudden and violent scream, complaining of acute pain in the bead, behind the left eye; the pain continued some minutes (two or three), during which time he uttered frequent screarns.

He was taken down stairs to the sofa by his mother, but finding bimself uncomfortable there, he walked into the next rom, and was assisted on to the bed. His mother ran out of the room for a glass of water, and upon her return found that he had fallen off the bed, and was completely insensible.

Drs. Rolph and Rankin were the first medical men who saw him; he 